\title{
LXIX. On some astringent substances as sources of pyrogallic acid
}

\section{Dr. John Stenhouse}

To cite this article: Dr. John Stenhouse (1843) LXIX. On some astringent substances as sources of pyrogallic acid , Philosophical Magazine Series 3, 22:147, 417-427, DOI: 10.1080/14786444308636411

To link to this article: http://dx.doi.org/10.1080/14786444308636411

$$
\text { 曲 Published online: } 01 \text { Jun } 2009 .
$$

Submit your article to this journal $[\pi$

Q View related articles $₫$ 
A N $\mathbf{D}$ JOURNAL OF SCIENCE.

\author{
[THIRD SERIES.]
}

$J U N E$ 184.3.

\title{
LXIX. On some Astringent Substances as Sources of Pyro-
} gallic Acid. By Dr. John Stenhouse.*

CHEMISTS have usually divided the varieties of tannin C which occur so abundantly in the vegetable kingdom, into two kinds; those which give black and those which give green precipitates with salts of iron. The propriety of this distinction has of late been called in question by Berzelius, who seems to think that the tannic acid in all plants is essentially the same; and that the green and gray colours of the precipitates with salts of iron are owing to the presence of free acid. Professor Liebig appears also to hold a similar opinion. Berzelius states at 116th of his Rapport Annuel for 1841 , on the authority of C. H. Cavallius, "that the tannic acids which give green precipitates with sulphate of iron, give blue precipitates with acetate of iron, and that their green combinations are rendered blue by the addition either of small quantities of acetate of lead, by a little alkali, or even by a great excess of gelatine." He also states that the lead compounds of those species of tannin which give green precipitates with salts of iron are rendered blue by the addition of a little sulphate of iron. He likewise affirms that when a solution of any of the tannins which give green precipitates remains in contact for some time with chips of iron, a blue instead of a green precipitate is obtained, and that its blue colour is changed to green by the addition of acetic acid. These statements have induced Berzelius to conclude that the giving green or black precipitates with salts of iron is not a distinctive character for any species of tannin, as these precipitates are convertible into each other; bases rendering them black or

- Communicated by the Chemical Society; having been read November $15,1842$.

Phil. Mag. S. 3. Vol. 22. No. 147. June 1843. 2 F 
blue, and acids gray or green. A few experiments which I have made have, however, given somewhat different results from those of Cavallius.

A solution of the tannin of catechu was prepared by macerating a large quantity of catechu in a very little cold water; it was therefore quite free from catechin. A portion of this solution was allowed to remain for some days in contact with a quantity of iron chips; it assumed a dirty, grayish black colour, which, however, did not at all resemble the blue-black colour which nut-galls, oak, bark, \&c. exhibit when similarly treated. The precipitate did not become green when a little acetic acid was added to it, but it dissolved in an excess of acid, and on being meutralized with ammonia a purple gray precipitate appeared. Tannin of catechu gave a dull grayish black precipitate with acetate of iron. With neutral acetate of lead it gave a light yellow precipitate, which on the addition of sulphate of iron assumed a dark gray colour, much lighter than the preceding. Basic acetate of lead gave similar results. Chloride of iron gave an olive green; perchloride an olive brown, and protonitrate a yellowish green precipitate. Tannin of catechu, when treated with acetic acid and sulphate of iron, gave a dark olive precipitate.

The tannin of larch bark, which gives a light green precipitate with protosulphate of iron, gave with acetate of iron a purplish black precipitate, which on standing for a day or so assumed a dark lead colour. When treated first with acetate of lead and then with sulphate of iron it gave a grayish purple precipitate. When mixed with a little acetic acid it gave a dark gray precipitate on the addition of sulphate of iron. Chloride of iron produces a grayish brown, and nitrate of iron an olive brown precipitate.

The tannin of gum kino gave with protosulphate of iron a dark green, and with acetate of iron a purplish black precipitate, which on standing changed to grayish black. With protochloride and protonitrate of iron dark green precipitates, which quickly changed to grayish brown. The effect of alkalies on all these precipitates was only to deepen their colours, but not to change any of them bluish black.

The tannin of alder bark, birch bark and tormentil root, gave purplish black precipitates with acetate of iron, which however on standing became grayish black, and their reactions with the inorganic salts of iron were almost identical with those of kino, catechu and larch bark, and wholly dissimilar with those of galls, sumach, valonia, \&c. Very good ink of a bluish black colour may be made from nut-galls, oak bark, sumach, valonia, divi-divi, \&c.; but the tannin of catechu, 
kino, larch, birch and alder barks, \&c., are wholly unfit for this purpose. Indeed the only salt of iron which gives pretty nearly the same coloured precipitates with either species of tannin is the acetate, but even these, though at first bluish black in the case of the green tannins, become in a day or two grayish black, and their reactions with the sulphate, chloride and nitrate of iron have no resemblance whatever to those of the black tannins. The old distinction, therefore, which divides the astringents into those which give black, and into those which give green precipitates with the inorganic salts of iron, appears, so far as it goes, to be perfectly just, as these precipitates are not convertible into each other, as affirmed by M. Cavallius. There is, however, good reason for believing that some of the varieties of tannin, which even agree in their reaction upon salts of iron, and in their characters generally, are still by no means identical substances. In this respect there is considerable analogy between the varieties of tannin and the different fatty acids.

It is much to be regretted that we are unable to procure tannin in a state of purity from any other source than nutgalls. When pounded galls are treated by Pelouze's method, with hydrated ather, in a displacement apparatus, the liquid on standing separates into two strata, the lowest of which contains tannic acid in a state of purity.

When, however, oak bark, valonia, sumach, gum kino, catechu, \&c. are treated with æther in a similar manner, only one stratum of liquid is obtained. Pelouze's process is therefore inapplicable to these substances. This is the more to be regretted, as from the extreme facility with which tannin decomposes when in contact with moisture, we are unacquainted with any good way of obtaining it in a pure state from any of the other astringent substances; and consequently, with the exception of nut-galls, little progress has been made in the investigation of these bodies.

It has been already mentioned that the tannin of galls and gallic acid are the only substances which, when distilled, are known to yield pyrogallic acid-a substance whose characters are so well marked that it can easily be recognized. It struck me, therefore, that this circumstance might be employed as an easy test for the presence of gallic acid; and also enable us to ascertain when the tannin these substances contained was similar or otherwise with that of nut-galls. With this view $I$ was induced to subject a number of the astringent matters to examination. The first in the order was sumach.

Sumach, which is so extensively employed in Great Britain $2 \mathrm{~F} 2$ 
by dyers and leather-curriers, consists of the small branches of the Rhus coriaria. A quantity of sumach was digested with hot water, filtered and evaporated to dryness. The dried extract thus obtained was subjected to distillation. The liquid which passed into the receiver, though it gave no crystals of pyrogallic acid, obviously contained that substance, as it exhibited all its characteristic reactions. The pyrogallic acid was prevented from crystallizing by the empyreumatic oil and other impurities by which it was accompanied. It seemed not improbable, therefore, that sumach contained gallic acid, and that perhaps its tannin was also similar to that of galls.

The first step taken, therefore, was to examine sumach for gallic. Several pounds of sumach were repeatedly boiled with water and then filtered. The tannin contained in the liquid was precipitated by solution of glue and separated by filtration. Its quantity was very considerable. The clear liquid was evaporated to the consistence of an extract, and treated with hot alcohol. The greater portion of the spirits was recovered by distillation, and the residue set aside to crystallize. After some days, as no crystals made their appearance, the alcoholic solution was evaporated to dryness on the waterbath. It was then introduced into a stoppered bottle and repeatedly agitated with æther; almost the whole of the æther was distilled off, and the residue left to spontaneous evaporation. Abundance of reddish coloured crystals soon appeared. They were purified by repeated digestions with animal charcoal and successive crystallizations. The crystals were then perfectly colourless and possessed the silky lustre of gallic acid, with which acid their reactions with salts of iron and other reagents completely corresponded. When distilled they yielded abundance of pyrogallic acid. They were dried at $212^{\circ} \mathrm{F}$. and subjected to analysis.

I. 0.2932 gramme substance gave 0.5315 carbonic acid, and 0.963 water.

II. 0.2824 gave 0.508 carbonic acid, and 0.956 water.

\begin{tabular}{crc} 
I. & \multicolumn{1}{c}{ II. } & Calculated. \\
C $50 \cdot 12$ & $49 \cdot 73$ & $7 \mathrm{C}=49 \cdot 89$ \\
H $3 \cdot 64$ & 3.76 & $3 \mathrm{H}=3 \cdot 49$ \\
O 46.24 & $\frac{46.51}{100 \cdot 00}$ & $5 \mathrm{O}=4.62$ \\
\hline $100 \cdot 00$ & $100 \cdot 00$
\end{tabular}

These results approach pretty closely the calculated numbers of hydrated gallic acid given above.

In order to determine the atomic weight of the acid, the basic gallate of lead was formed by adding a solution of the 
Astringent Substances as Sources of Pyrogallic Acid. 4.21

acid obtained from sumach to an excess of boiling acetate of lead. It precipitated as a yellow, slightly crystalline powder, and was also dried at $212^{\circ} \mathrm{F}$.

I. 0.803 salt gave 0.4 .56 oxide and 0.14 .2 metallic lead $=$ $75 \cdot 84$ per cent. oxide of lead.

II. 0.6972 gave 0.332 oxide and 0.186 metallic lead $=76.35$ oxide.

Now the bibasic gallate of lead $\mathrm{C}^{7} \mathrm{H} \mathrm{O}^{3}+2 \mathrm{~Pb} \mathrm{O}$ contains $76^{\circ} 69$ per cent. oxide of lead; therefore there can be no doubt that it was the salt analysed, and that gallic acid, therefore, occurs to a considerable extent ready formed in sumach.

I next proceeded to examine the tannin. In order to obtain the tannin of sumach free from gallic acid, I macerated a very considerable quantity of sumach in a very little cold water, filtered the liquid, and threw down the tannin it contained by adding to the solution about half its bulk of sulphuric acid by small quantities at a time. The precipitate, which had a brownish yellow colour, was tolerably abundant. It was collected on a cloth filter, washed with a little cold water, and strongly compressed so as to remove as much of the sulphuric acid as possible. It was then dried and distilled. It yielded crystals of pyrogallic acid as freely as the same quantity of tannin of galls would have done. The products of the distillation of tannin from both these sources are, therefore, the same.

In order to see if the analogy extended any further, I was induced to try if the tannin of sumach would be converted into gallic acid by being boiled with dilute sulphuric acid, as is the case with tannin when similarly treated. A second portion, therefore, of the tannin of sumach precipitated by sulphuric acid and purified like the former, was boiled for an hour with a mixture of two parts water and one of sulphuric acid. It was filtered while hot. When the liquid cooled, an abundant crop of hard, dark brown crystals appeared. They were collected on a filter and washed with a little cold water, then pressed and dried. They were repeatedly dissolved in small quantities of water, and boiled with purified animal charcoal till they were perfectly colourless. When subjected to analysis,

I. 0.279 acid gave 0.505 carbonic acid, and 0.945 water. II. 0.314 .5 gave 0.5675 carbonic acid, and 0.1056 water.

\begin{tabular}{crr} 
I. & \multicolumn{1}{c}{ II. } & Calculated. \\
C 50.04 & 49.78 & $7 \mathrm{C}=49.89$ \\
$\mathrm{H} 3.76$ & 3.73 & $3 \mathrm{H}=3.4 .9$ \\
$\mathrm{O} 46.20$ & 46.49 & $5 \mathrm{O}=4.6 .62$ \\
\hline 100.00 & 100.00 &
\end{tabular}


These results again agree with the numbers of hydrated gallic acid.

The bibasic gallate of lead was also formed with another portion of the acid in the way already described.

I. $0 \cdot 690$ salt gave $0 \cdot 153$ oxide and $0 \cdot 3448$ lead $=76 \cdot 18$ per cent. oxide.

II. $0 \cdot 74.15$ salt gave $0 \cdot 1745$ oxide and 0.3635 lead $=76 \cdot 34$ per cent. oxide.

There can be no doubt, therefore, that the acid produced by the action of sulphuric acid on the tannin of sumach is the gallic acid, precisely as is the case with the tannin of galls. Another portion of the tannin of sumach precipitated also by sulphuric acid, and which had been freed as much as possible, by being washed and compressed, from any adhering acid, was kept well moistened in an open vessel for more than five weeks at a temperature of about $70^{\circ} \mathrm{F}$. It readily yielded crystals of gallic acid when treated with alcohol and æether in the way already described. Sumach, therefore, appears to approach the nature of nut-galls more closely than any of the other astringent substances. This fact is well known to 'Turkey-red dyers, who have long successfully employed sumach as a substitute for galls; as might be expected, a greater weight of sumach is required to produce the same effect, as the quantity of gallic acid and tannin contained in sumach is much less in proportion to its bulk than in nut-galls.

The effect of the action of sulphuric acid upon the tannin, both of sumach and of galls, varies very much according to the strength of the acid employed. When the preparation of gallic acid alone is our object, we succeed best by using acid diluted with seven or eight times its bulk of water. The digestion should be continued for a day or so, new quantities of water being added from time to time as the evaporation proceeds. When the digestion is finished, the liquid should be concentrated by a very gentle heat. Nearly the whole of the tannin is converted into gallic acid, which is deposited in crystals which are only slightly coloured, and therefore easily purified. If, on the other hand, more concentrated acid is used, the crystals are very dark-coloured, and require repeated digestion with animal charcoal, which occasions both trouble and loss. Besides, concentrated acid converts only about a half of the tannin into gallic acid. The other portion is changed into a dark-coloured pulverulent substance possessing decided acid properties, and very much resembling humus in appearance.

Action of Muriatic Acid upon Tannin.

Muriatic acid converts tannin into gallic acid in precisely 
the same way as sulphuric acid does. If the muriatic acid is pretty dilute, and the digestion conducted with a moderate heat, the tannin is almost wholly resolved into slightly coloured gallic acid, and a very little only of the insoluble black matter appears. If, on the contrary, we employ an acid diluted with even three times its bulk of water, and continue the boiling for an hour, about half of the tannin is changed into very dark coloured gallic acid, and the rest is converted into the insoluble black matter already mentioned. In order to ascertain that the acid obtained by this process was really gallic acid, it was purified and subjected to analysis. It possessed all the reactions of ordinary gallic acid.

I. 0.2721 gramme substance gave 0.487 carbonic acid, and 0.0865 water.

II. 0.2902 gave 0.5235 carbonic acid, and 0.091 water.

\begin{tabular}{crc} 
I. & II. & Calculated. \\
C $49 \cdot 49$ & 49.87 & $7 \mathrm{C}=49 \cdot 89$ \\
H 3.53 & 3.48 & $3 \mathrm{H}=3.49$ \\
O 46.98 & $\frac{46.65}{100 \cdot 00}$ & $5 \mathrm{O}=4.62$ \\
\hline $100 \cdot 00$ & &
\end{tabular}

In order to determine the atomic weight of the acid its basic lead salt was prepared in the way already mentioned.

I. 0.758 salt gave 0.245 oxide and 0.307 metallic lead $=75.95$ per cent. oxide of lead.

II. 0.771 salt gave 0.212 oxide and 0.347 metallic lead $=$ $75 \cdot 97$ oxide per cent.

The calculated quantity of oxide of lead in the bibasic gallate is 76.69 per cent.

From these results there can be no doubt that the acid was gallic acid.

Tannin is precipitated from its solution by muriatic acid even more perfectly than by sulphuric acid, and it is a matter of indifference which of the two acids we employ in order to convert it into gallic acid. The only advantage attending the employment of muriatic acid is that by evaporating the mixture of the two acids nearly to dryness on the water-bath, the greater portion of the muriatic acid may be easily driven off.

Nitric acid does not precipitate tannin from its solution, but almost immediately converts it with evolution of deutoxide of azote into very pure oxalic acid.

The pulverulent substance already mentioned as produced along with gallic acid by the action of muriatic and sulphuric acids upon tannin, has very much the colour of soot and is nearly tasteless. It is insoluble in cold water, and very slightly so in boiling water. When laid on moistened litmus paper, 
however, it reddens it strongly. It consists of at least two substances; one of which only is soluble in hot alcohol, so that they may be easily separated by this means. They both dissolve very readily in alkalies, and decompose the carbonates when assisted by heat. When saturated with ammonia and rendered neutral by digestion, they give dark brown precipitates with salts of silver, copper, iron, lead, barytes and lime. Their alkaline solutions are dark brown, and they are completely precipitated by acids. These humus-like substances are produced by the action of the acids on the tannin only, for on boiling gallic acid with concentrated muriatic acid for several hours $I$ did not succeed in obtaining even a trace of them. I am at present engaged in their further investigation.

Valonia.-.'The next of the astringent matters examined was valonia. It is the acorn of the Quercus AEgilops, and is imported in considerable quantities from the Levant for the use of the tanners. The dried extract of valonia prepared like that of sumach, when destructively distilled, gave no indication of pyrogallic acid. Valonia was next examined for gallic acid. A strong solution of it was precipitated by glue, and the clear liquid evaporated to an extract and heated with spirits of wine. The spirits of wine were distilled off, and the residue treated with ather exactly as sumach had been. A very small quantity of crystals was obtained. They exhibited the reactions of gallic acid on salts of iron and other reagents, and when distilled yielded crystals of pyrogallic acid. I have every reason to believe that these crystals were gallic acid, though from the smallness of their quantity I was unable to subject them to analysis. Valonia, therefore, may be regarded as containing a little gallic acid, but its quantity is so inconsiderable as probably not to amount to a thirtieth of what sumach contains.

The most concentrated solutions of valonia give a very scanty precipitate when treated with sulphuric acid; and a large quantity of valonia must therefore be employed to yield any quantity of tannin by this process. The precipitate has a bright yellow colour. When distilled it left a very bulky charcoal, and gave scarcely any empyreumatic products. The liquid which passed into the receiver was nearly colourless, and did not give the least indication of pyrogallic acid. The tannin of valonia appears, therefore, essentially different from that of nut-galls.

Oak Bark.-The extract of oak bark when dried and distilled, also gave no indication of pyrogallic acid. I then endeavoured to obtain gallic acid by treating a decoction of oak bark in the way already described. Though I operated on 
considerable quantities, such as six and eight pounds, I did not succeed in the course of several trials in obtaining any crystals of gallic acid. I apprehend, therefore, that if oak bark contains any gallic acid at all, it must exist in very minute quantity. The tannin of oak bark when precipitated by sulphuric acid had a reddish brown colour. When subjected to distillation it gave no indication of pyrogallic acid. The tannin was also boiled with dilute sulphuric acid. It became darker coloured and nearly insoluble either in hot or cold water. It was a little more soluble, though very slightly so, in alkaline leys. On the addition of an acid a few reddish flocks precipitated. Spirits of wine also dissolved a little of it, and assumed a light red colour. The tannin of oak bark appears, therefore, also to differ from that of nut-galls.

Divi-diri.-The astringent substance by some called Dividivi, by others Libi-divi, has of late years been imported into Great Britain from Carthagena in considerable quantity. It is the pod of a leguminous shrub which grows to the height of between twenty and thirty feet. Professor Balfour informs me that its botanical name is Casalpin coriaria. It is a native of South America, and is noticed by Dr. McFadyen in his Flora of Jamaica, as occurring in that island. The pods of this shrub, which form the divi-divi of commerce, are of a dark brown colour, nearly three inches long and about half an inch broad. They are very much curled up, as if they had been strongly dried; and contain a few flattish seeds. The taste of divi-divi is highly astringent and bitter. The astringent matter is contained only in the outer rind of the pod; the inner skin, which incloses the seeds, is nearly tasteless. The pods are often perforated with small holes, evidently the work of some insect. The aqueous solution of divi-divi gives a copious precipitate with gelatine, and strikes a deep blue with persalts of iron.

When dried extract of divi-divi-was distilled, the liquid which passed into the receiver, though it gave no crystals of pyrogallic acid, evidently contained that substance, as it exhibited all its characteristic reactions.

When divi-divi was treated for gallic acid in the way $\mathbf{I}$ have already described, I easily succeeded in obtaining a considerable quantity of reddish-coloured crystals, which, when purified like the others with animal charcoal, became perfectly white. They had the usual reactions of gallic acid, and yielded pyrogallic acid when distilled.

When dried at $212^{\circ} \mathrm{F}$. and subjected to analysis,-

I. 0.3034 gramme acid gave 0.550 carbonic acid, and 0.1018 water. 
II. 0.3052 gave 0.5505 carbonic acid and 0.1012 water.
I.
II.
Calculated.
C $50 \cdot 12$
$49 \cdot 87$
$7 \mathrm{C}=49 \cdot 89$
H 3.72
$3 \cdot 71$
O $46 \cdot 16$
$46 \cdot 42$
$\overline{100 \cdot 00}$
$100 \cdot 00$
$3 \mathrm{H}=3 \cdot 49$
$5 \mathrm{O}=4.6 \cdot 62$

In order to determine the atomic weight of the acid, I formed the bibasic gallate of lead.

I. 0.706 of this salt gave 0.3325 lead, and 0.1802 oxide $=$ 76.25 per cent. oxide of lead.

II. 0.887 salt gave 0.315 lead and 0.340 oxide of lead $=$ 76.58 per cent oxide.

Now the bibasic gallate of lead $\mathrm{C}^{7} \mathrm{H} \mathrm{O}^{3}+2 \mathrm{P}$ bo contains 76.69 per cent oxide of lead; there can, therefore, be no doubt that it was the salt analysed, and that gallic acid occurs to a considerable extent ready formed in divi-divi.

Sulphuric acid throws down a very scanty dark brown precipitate, even in highly concentrated solutions of divi-divi. When dried and distilled, it did not yield any trace of pyrogallic acid, and left a very bulky charcoal. The tannin of divi-divi appears, therefore, essentially different from that of nut-galls.

Mr. Harvey informs me that a few years ago some calicoprinters endeavoured to employ divi-divi as a substitute for galls, but the large quantity of mucilage it contained rendered it unfit for this purpose.

It is at present pretty extensively employed in the tanning of leather, as the quantity of tannin it contains is considerable, and the presence of mucilage is not injurious to that process.

Gum Kino.-The species of kino which I examined was the African variety. I was unable to detect in it any trace of gallic acid. Sulphuric acid threw down the tannin of kino as a bulky dark red precipitate. When distilled, it gave scarcely any volatile products, and no trace of pyrogallic acid. When digested with nitric acid, gum kino was wholly converted into oxalic acid.

Catechu.-It was the light-coloured cubical variety of catechu that I employed. It does not contain any gallic acid, but catechin and a variety of tannin which gives olive green precipitates with salts of iron. Sulphuric acid throws down the tannin of a brownish yellow colour. When boiled with dilute sulphuric acid, it becomes dark brown. Like the tannin of oak bark, it is insoluble either in cold or boiling water. When boiled in strong alkaline solutions, it only dissolves to a very small extent. It is also insoluble in alcohol and æther. 
When distilled, the tannin gave no indication either of pyrogallic acid or of pyrocatechin.

Catechin, which is the part of catechu insoluble in cold water, when distilled, yielded the pyrocatechin of $Z$ wenger in considerable quantity. So far as I examined it, pyrocatechin appeared to possess the properties ascribed to it by that chemist.

In conclusion, I may mention that this is only the first of a series of papers on the astringent substances.

Glasgow, Oct. 26, 1842.

LXX. On some nere Cases of Voltaic Action, and on the Construction of a Battery roithout the use of Oxidizable Metals. By Alexander R. Arrott, Esq**

HAVING been for some time engaged in examining into certain remarkable voltaic actions occurring in cases hitherto unobserved, or at least not followed out so fully as their importance seems to demand, I am induced brietly to communicate the results of my inquiries, hoping they may not prove uninteresting to the Society.

It is a fact known to every one who has carefully observed the phænomena attending chemical decomposition by means of electricity, that the electrodes immersed in the solution undergoing decomposition, acquire the power of producing a current in the opposite direction to that previously passing through them, when they are made to touch each other without being removed from the liquid.

This effect has generally been ascribed to a power supposed to be acquired by the metals, of producing a current independently of any action of the liquids beyond that of simply completing the circuit, and has been called "polarization of the electrodes."

Becquerel was the first who advanced the opinion, that the effect was due to the alteration produced in the liquid by the current causing decomposition. He supposed that the current was a consequence of the combination of the acid and alkaline produced at the positive and negative surfaces; but it will be found that the most powerful acids and alkalies are incapable of producing a current, unless they readily undergo some other change than that which takes place when an acid and alkali combine as such. It has often been shown, that sulphuric acid and potash, for example, are nearly or altogether

* Communicated by the Chemical Society; having been read November 15,1842 . 